\title{
Higher-order metaphysics and the tropes versus universals dispute
}

\author{
Lukas Skiba ${ }^{1}$
}

Accepted: 23 October 2020/Published online: 25 November 2020

(C) The Author(s) 2020

\begin{abstract}
Higher-order realists about properties express their view that there are properties with the help of higher-order rather than first-order quantifiers. They claim two types of advantages for this way of formulating property realism. First, certain gridlocked debates about the nature of properties, such as the immanentism versus transcendentalism dispute, are taken to be dissolved (roughly: avoided). Second, a further such debate, the tropes versus universals dispute, is taken to be resolved (roughly: decided). In this paper I first argue that higher-order realism does not in fact resolve the tropes versus universals dispute. In a constructive spirit, I then develop higher-order realism in a way that leads to a dissolution, rather than a resolution, of this dispute too.
\end{abstract}

Keywords Higher-order metaphysics · Properties · Tropes · Universals · Higherorder logic $\cdot$ Higher-order quantification

\section{Higher-order metaphysics and property theories}

A foundational question in metaphysics is what (formal) language metaphysical theorizing should be conducted in. An increasingly popular response is provided by higher-orderism. Higher-orderists insist that metaphysical theories should be formulated in a language that allows not only for first-order quantification, i.e. quantification into the position of singular terms, but also for sui generis higherorder quantification, i.e. quantification into other syntactic positions which is not reducible to or explicable in first-order terms.

Lukas Skiba

lukas.skiba@uni-hamburg.de

1 Philosophisches Seminar, Universität Hamburg, Überseering 35, Postfach \#4, 22297 Hamburg, Germany 
In this spirit, higher-order resources have recently been brought to bear on a diverse list of topics, including unrestricted quantification (Williamson 2003), essence (Correia 2006), the internalism versus externalism debate (Besson 2009), the contingentism versus necessitism debate (Stalnaker 2012; Williamson 2013; Fritz and Goodman 2017), generalized identity (Rayo 2013; Dorr 2016), propositions (Trueman 2018, 2020; Jones 2019) and grounding (Correia and Skiles 2019).

A further and particularly natural focal point for higher-orderism is the metaphysics of properties. After all, properties are intimately connected to predicates and quantification into predicate position constitutes the paradigm case of higher-order quantification. Exploiting this connection, Jones (2018) and Trueman (forthcoming) argue that property realism finds its natural habitat in a higher-order, rather than a first-order, setting. To bring out what is at issue here, we can follow Jones (809-811) in taking proponents of first-order realism about properties (FOR) to endorse a range of substitution instances of the following schema:

(Exist1) $\square \forall x(\Phi x \rightarrow \exists y(I x y \wedge \square \forall z(I z y \rightarrow \Phi z)))$

Here ' $I x y$ ' is to be read as ' $x$ instantiates $y$ ' so that replacing the schematic letter ' $\Phi$ ' with, say, the predicate ' $N$ ' (to be read as '...is negatively charged') yields the claim that, necessarily, for each negatively charged object there is something which it instantiates and, necessarily, only negatively charged objects instantiate that thing:

$$
\square \forall x(N x \rightarrow \exists y(I x y \wedge \square \forall z(I z y \rightarrow N z)))
$$

The properties countenanced by FOR are thus first-order entities: they are the witnesses for the first-order existential quantifier in instances of (Exist1).

Contrast this with second-order realism about properties (SOR) whose proponents instead assert instances of the following schema:

$$
\square \forall x(\Phi x \rightarrow \exists Y(Y x \wedge \square \forall z(Y z \rightarrow \Phi z)))
$$

Here the first-order existential quantifier has made way to a second-order existential quantifier that binds variables in the position of predicates (more-precisely: firstlevel predicates, such as ' $N$ '). The properties countenanced by SOR are thus secondorder entities: they are the witnesses for the second-order existential quantifier in instances of (Exist2). ${ }^{1}$

Why should we think of properties as second-order rather than first-order entities? The higher-orderists' overriding claim is that this results in a theoretically rewarding transformation of an all too well-trodden metaphysical territory. The transformation is two-fold: first, SOR is taken to dissolve (roughly: avoid) certain

\footnotetext{
1 Three more comments on (Exist1) and (Exist2): First, the schemas are designed to be acceptable to proponents of universals as well as to trope theorists. This is why the second conditional is not strengthened to a biconditional. Second, my presentation differs from Jones in that he considers schemas covering predicates of any arity. For simplicity, I focus mainly on the monadic case throughout this paper. Third, like Jones, I focus mainly on the second-order portion of the higher-orderists' view on properties, while acknowledging that they will make analogous claims on all other higher-order levels (see Sects. 4 and 6).
} 
intractable disputes regarding the nature of properties, such as the immanentism versus transcendentalism dispute. Second, SOR is taken to resolve (roughly: decide) a further such dispute, namely the tropes versus universals dispute.

While I agree that a higher-order conception of properties leads to a fruitful reshaping of a familiar metaphysical landscape, I come to a different conclusion about the result of this shape-shift: after some brief stage-setting (Sect. 2), I argue that SOR does not, in fact, resolve the dispute between tropes and universals (Sects. 3 and 4). In a constructive spirit, I then provide a new way of motivating the use of higher-order resources in property theories: I develop and defend hybrid realism, a position that combines elements of FOR and SOR to effect a dissolution rather than a resolution of the tropes versus universals dispute (Sects. 5 and 6).

\section{Arguments for second-order realism}

According to the argument from dissolution, SOR dissolves certain intractable disputes regarding the nature of properties. A representative example is the immanentism versus transcendentalism dispute, understood as the dispute over the question whether properties are spatiotemporally located (see Jones 2018: $\S 4){ }^{2}$ In brief, the idea is that SOR reveals this dispute to be based on a false dilemma: it is based on the assumption that any property realist has to answer the question of whether properties are located either positively or negatively. SOR, however, takes properties to be entities in the range of sui generis second-order quantifiers, quantifiers binding variables in the syntactic positions of predicates. Accordingly, SOR regards predicates, rather than singular terms, as the linguistic entities suitable to facilitate talk about properties. But locational vocabulary, such as '... is located at-', is most naturally construed as combinable only with singular terms. Therefore, the argument from dissolution concludes, second-order realists are entitled to refuse the question after the location of properties as unintelligible, thereby evading the need to engage with it.

According to the argument from resolution, SOR resolves the tropes versus universals dispute, understood as the dispute over the question whether properties are repeatable (see Jones 2018: §5). Tropes are taken to be unrepeatable entities: no (monadic) trope can be instantiated by more than one object. Therefore, first-order trope theory is taken to be characterized by the combination of (Exist1) with:

(Trope1) $\square \forall x \forall y(I x y \rightarrow \square \forall z(z \neq x \rightarrow \neg I z y))$

Universals are repeatable entities: (monadic) universals typically can be instantiated by more than one object. Therefore, first-order universal theory is taken to be characterized by the combination of (Exist1) with (suitable instances of):

(Universal1) $\exists x \square \forall y(I y x \leftrightarrow \Phi y)$

\footnotetext{
${ }^{2}$ For further instances of this argumentative strategy see Trueman (forthcoming: Ch. 10).
} 
(Trope1) and (Universal1) conflict with one another. Thus an instance of (Universal1) tells us that there is a thing which necessarily all and only negatively charged things instantiate. In particular, this negatively charged electron and that negatively charged electron instantiate it. But (Trope1) entails that whatever this electron instantiates cannot be instantiated by that electron. So given that there are two negatively charged electrons, (Trope1) and (the relevant instance of) (Universal1) cannot both be true. First-order realists thus face a choice between trope theory and universal theory. But how are they to choose? According to the argument from resolution, the debate between first-order trope theory and first-order universal theory has become bogged down in a stalemate that leaves no reasonable hope to be resolved any time soon. Granted this assumption, it would appear to be an advantage of SOR if it doesn't result in a similar gridlock. And, according to Jones, it doesn't. For second-order realists will not have to choose between (Trope1) and (Universal1) but between their second-order variants:

$$
\begin{aligned}
& \text { (Trope2) } \square \forall x \forall Y(Y x \rightarrow \square \forall z(z \neq x \rightarrow \neg Y z)) \\
& \text { (Universal2) } \quad \exists X \square \forall y(X y \leftrightarrow \Phi y)
\end{aligned}
$$

The crucial claim of the argument from resolution is now that while the first-order realist's choice between (Trope1) and (Universal1) is hard, the second-order realist's choice between (Trope2) and (Universal2) is easily resolved in favor of the latter. Two arguments are provided in support of this.

The comprehension argument First, Jones maintains that, in a second-order setting, any instance of the universalist comprehension schema (Universal2) is logically derivable from an uncontroversial truth. Thus consider an arbitrary instance of (Universal2):

$$
\exists X \square \forall y(X y \leftrightarrow F y)
$$

And consider the corresponding uncontroversial truth:

$$
\square \forall y(F y \leftrightarrow F y)
$$

Jones points out that (2) can be derived from (3) by application of a rule for secondorder existential generalization (modeled on the rule for classical first-order existential generalization) which we can put as follows (cf. 2018: 812):

(EG2) From $A(\Phi)$ one may infer $\left\ulcorner\exists X A^{[X / \Phi]}\right\urcorner$ where $A^{[X / \Phi]}$ results from $A(\Phi)$ by replacing (zero or more) occurrences of the predicate $\Phi$ with ' $X$ '.

Since Jones takes (EG2) to be part of the background second-order logic, he concludes that in a second-order setting, there is a logical argument for universalism.

The same type argument Second, Jones takes (Trope2) to conflict with what he regards as 'one of the most basic aspects of our ordinary and scientific world-views' namely that 'many distinct objects can be of the same type' (826). For a property

\footnotetext{
${ }^{3}$ For brevity, I suppress here and in the following quantifier rules the standard conditions on ' $X$ ' namely that (a) $\Phi$ is free for ' $X$ ' in $A$ and (b) $\Phi$ and ' $X$ ' are of the same arity.
} 
theory to allow for this phenomenon, Jones argues, it must allow for the truth of sentences that have the following form:

(Same Type) $\Phi a \wedge \Phi b \wedge a \neq b$

For example, in order to allow for the fact that electron $a$ is distinct from electron $b$ but they are both negatively charged, a property theory must allow for the truth of ' $N a \wedge N b \wedge a \neq b$ '. But, the argument concludes, second-order trope theory does not allow for any such truths, since (Trope2) entails any sentence of the following form:

(4) $(\Phi a \wedge a \neq b) \rightarrow \neg \Phi b$

To consider the example again, (Trope2) entails ' $(N a \wedge a \neq b) \rightarrow \neg N b$ ', thereby ruling out the possibility of there being two negatively charged objects.

At first sight, the argument from resolution for SOR may seem entirely convincing. Both the comprehension argument and the same type argument seem initially appealing. And, importantly, neither has an analogue on the first-order level. Instances of (Universal1) can't be derived from uncontroversial truths by firstorder existential generalization. And (Trope1) doesn't conflict with (Same Type). So it may indeed look as if the dispute between (Trope2) and (Universal2) was quickly resolved in favor of the latter and as if this resolution was genuinely attributable to the second-order framework in which the competing theories are formulated.

On closer inspection, however, the argument from resolution will emerge as unsuccessful. This is because there is a natural alternative way of setting up secondorder trope theory which will render it immune to both the comprehension argument and the same type argument. I will describe this alternative set up in Sect. 3. In doing so, it is important to keep in mind the dialectical purpose of this endeavor. The aim is not to show that the resulting second-order trope theory is just as good, let alone superior to, second-order universal theory. Rather, the aim is to show that a certain way of arguing for SOR - the argument from resolution-is unsuccessful.

\section{Tropes versus universals: dispute resolved?}

We saw that the comprehension argument explicitly relies on a classical rule of second-order existential generalization. The first thing to note is that the same type argument implicitly relies on a corresponding rule for universal instantiation. Thus sentences of the form (4) can only be derived from (Trope2) given a rule such as:

(UI2) From $\ulcorner\forall X A(X)\urcorner$ one may infer $A^{[\Phi / X]}$ which results from $\ulcorner A(X)\urcorner$ by replacing all occurrences of the variable ' $X$ ' with the predicate $\Phi$.

So Jones' arguments against higher-order trope theory have a common source in the classical rules for the second-order quantifiers he presupposes. This raises the prospect of developing a more sophisticated version of second-order trope theory in a setting that supports alternative quantifier rules. Note in particular that classical 
quantifier rules are in fact quite controversial once we're operating in a modal setting. For in such a setting, they swiftly lead to the controversial conclusion that the entities they quantify over exist necessarily (more on this below). Since a prominent option to avoid such results is to employ free quantifier rules instead, a natural suggestion is to develop second-order trope theory in a free setting. After noting further reasons for second-order trope theorists to forgo classical secondorder quantifiers (Sect. 3.1), a free version of second-order trope theory is developed (Sect. 3.2) and shown to be immune to Jones' arguments (Sect. 3.3). The upshot is that the argument from resolution fails (Sect. 3.4).

\subsection{Second-order trope theory and classical quantifiers}

Independently of Jones' arguments, there are at least three reasons why secondorder trope theory doesn't sit well with classical second-order quantifiers.

Sparse tropes Many who think of properties as tropes favor sparse theories of properties (see e.g. Ehring 2011). That is, they don't take each and every predicate to correspond to a property, but only some special subclass, for instance those that are sufficiently natural in the sense of Lewis (1983). They may accept, e.g., mass, charge and spin tropes but reject tropes of being polka-dotted, living next to a park, or having recently had eggs for breakfast. But the classical rules for second-order quantifiers naturally lead to an abundant conception of properties, according to which each predicate, natural or not, corresponds to a property. Thus the following sentence is uncontroversial, for any predicate ' $F$ ' whatsoever:

$$
\square \forall x(F x \rightarrow(F x \wedge \square \forall z(F z \rightarrow F z)))
$$

But, given (EG2), we can deduce from this the corresponding instance of (Exist2):

$$
\square \forall x(F x \rightarrow \exists Y(Y x \wedge \square \forall z(Y z \rightarrow F z)))
$$

So, given (EG2), even if ' $F$ ' expresses the most unnaturally gerrymandered condition imaginable, the assumption that, say, $a$ is $F$ forces us to ascribe to $a$ a corresponding second-order property of $F$-ness.

In fact, in the presence of (EG2), even predicates that don't apply to anything will bring about an existential commitment. For, presumably, the second-order analogues of first-order self-identity statements will be true for any predicate, even uninstantiated ones ${ }^{4}$ :

(7) $F \approx F$

(EG2) then allows us to infer:

$$
\text { (8) } \exists X(X \approx F)
$$

\footnotetext{
${ }^{4}$ Second-order trope theorists will most likely take second-order identity ' $X \approx Y$ ' as primitive or interpret it as higher-order indiscernability: $\forall Z^{2}\left(Z^{2} X \leftrightarrow Z^{2} Y\right.$ ) (where ' $Z^{2}$ ' is a third-order variable occupying the same syntactic position as monadic second-level predicates). Interpretations that result in less fine-grained individuation conditions for properties, such as necessary co-intensiveness $(\square \forall x \square(X x \leftrightarrow Y x)$ ), are inappropriate for their purposes since they will e.g. preclude an object from having more than one trope essentially.
} 
So given the classical quantifier rules there is no predicate at all that doesn't carry an existential commitment to a second-order entity. The classical second-order quantifier rules thus conflict with sparse conceptions of second-order tropes. ${ }^{5}$

No free floating tropes It is not only part of standard conceptions of tropes that they cannot have more than one bearer (as encoded by (Trope2)) but also that they cannot have less than one bearer. The most natural way to express such a ban on free floating tropes in a second-order framework is:

(NFF) $\square \forall X \exists y X y$

But, given the classical rule (UI2), this results in a commitment to ' $\exists y F y$ ' for any predicate ' $F$ ' whatsoever. Hence the attempt to ban flee floating tropes leads to the absurd view that for any condition that can be expressed by a predicate there is some object that fulfills the condition. The classical second-order quantifier rules thus conflict with a natural way of banning free-floating second-order tropes.

Contingent tropes Trope theorists are in trouble if they have to regard all tropes as existing necessarily. For given that tropes can't swap bearers and can't be bearerless, no trope can exist without being borne by its actual bearer. The necessary existence of the apple's redness trope, say, thus implies the necessary existence of the apple. If all objects have tropes, necessitism about tropes thus implies necessitism about all objects. While such a necessitism has struck many philosophers as absurd, it has recently been forcefully defended by Williamson (2013). Crucially, though, trope theorists face an even more radical conclusion: given that the redness trope is essentially a redness trope, its necessary existence implies that the apple is necessarily red. If all objects have tropes, necessitism about tropes not only implies that all objects exist necessarily, but that all objects necessarily are the way they actually are. Since this is clearly unacceptable, those who take properties to be tropes are under particular pressure to be contingentists about properties. Given natural background assumptions, however, classical rules for second-order quantifiers lead to necessitism about second-order properties: plausibly, second-order self-identity statements, e.g. ' $F \approx F$ ', will not only be true but logically true, just like first-order self-identities, e.g. ' $a=a$ ', are. Given (EG2), ' $\exists X(X \approx F)$ ' is then also a logical truth. A standard rule of necessitation (according to which all logical truths hold necessarily) then yields the necessitist claim:

(NEC) $\square \exists X(X \approx F)$

The classical second-order quantifier rules thus conflict with second-order tropes being contingent.

\footnotetext{
5 To this problem Jones suggests a solution (813) distinct from the one I advocate below: trope theorists are advised to accept that all predicates correspond to tropes and to preserve their sparse conception of properties by declaring many of these tropes as unnatural. One problem with this strategy is that trope theorists who are opposed to the very existence of tropes such as that of recently having had eggs for breakfast will not be helped much by concessions concerning their classification. In any case, this strategy won't help with the other two problems.
} 


\subsection{Second-order trope theory and free quantifiers}

Second-order trope theorists can avoid these problems by adopting a logic that allows predications to be free of existential commitments with respect to their predicative component. While free logics are most familiar in a first-order setting, where they allow predications to be free of existential commitments with respect to their singular term component, the crucial ideas are naturally extended to the second-order case (see Besson 2009; Bacon et al. 2016).

Thus free first-order logic allows singular terms to be non-denoting. And positive free first-order logic additionally allows atomic predications to be true even when they involve such non-denoting singular terms. In such a setting, the claim that Bilbo is a hobbit ' $H b$ ' entails the existence of hobbits ' $\exists x H x$ ' only in conjunction with an additional assumption to the effect that the relevant term denotes, i.e. ' $\exists x(x=b)$ '. In general, free first-order existential generalization doesn't allow us to derive $\ulcorner\exists x(A(x))\urcorner$ from $A(t)$ alone, but only from $A(t)$ and $\ulcorner\exists x(x=t)\urcorner$.

Analogously, free second-order logic allows predicates to be non-denoting. And positive free second-order logic additionally allows atomic predications to be true even when they involve such non-denoting predicates. In such a setting, the claim that Olga recently had eggs for breakfast ' $B o$ ' entails the existence of a property which Olga has, ' $\exists X(X o)^{\prime}$, only in conjunction with an additional assumption to the effect that the relevant predicate denotes, i.e. ' $\exists X(X \approx B)$ '. In general, free secondorder existential generalization is weakened in analogy to free first-order existential generalization and can be put as follows:

$\left(\mathrm{EG} 2_{F}\right) \quad$ From $A(\Phi)$ and $\ulcorner\exists X(X \approx \Phi)\urcorner$ one may infer $\left\ulcorner\exists X A^{[X / \Phi]}\right\urcorner$ where $A^{[X / \Phi]}$ results from $A(\Phi)$ by replacing (zero or more) occurrences of the predicate $\Phi$ with ' $X$ '.

The corresponding free rule for second-order universal instantiation is:

$\left(\right.$ UI2 $\left._{F}\right) \quad$ From $\ulcorner\forall X A(X)\urcorner$ and $\ulcorner\exists X(X \approx \Phi)\urcorner$ one may infer $A^{[\Phi / X]}$ which results from $\ulcorner A(X)\urcorner$ by replacing all occurrences of the variable ' $X$ ' with the predicate $\Phi$.

In a positive free setting, second-order trope theorists can develop their theory in a way that will allow them to both avoid the problems identified in Sect. 3.1 as well as to address Jones' arguments. The crucial move is to mark out a special class of predicates certain specimens of which are to stand for tropes and to regard the remaining predicates as non-denoting. Thus let our trope theorists introduce, for each pair of an ordinary predicate $\Phi$ and a term $t$, a new predicate $\Phi_{t}$ (the particularization of $\Phi$ to $t$ ) which is subject to the stipulation that, necessarily, for any $x, \Phi_{t}$ applies to $x$ just in case $\Phi$ applies to $x$ and $\ulcorner x=t\urcorner$ is true.

To illustrate, consider the ordinary predicate '... is negatively charged' (' $N$ ') and suppose that the electrons $a$ and $b$ are the only negatively charged objects. While the ordinary predicate ' $N$ ' applies to $a$ and $b$, the particularized predicate ' $N_{a}$ ' applies only to $a$, the particularized predicate ' $N_{b}$ ' applies only to $b$, and the particularized predicate ' $N_{c}$ ' doesn't apply to anything, since $c$ is not among the negatively charged objects. 
The sentences ' $N a$ ' and ' $N b$ ' say, correctly, that $a$ and $b$ are negatively charged, ' $N_{a} a$ ' and ' $N_{b} b$ ' ascribe, also correctly, to $a$ to $b$ their particularized negative charge, while ' $N_{a} b$ ' and ' $N_{b} a$ ' incorrectly ascribe to $a$ and $b$ the negative charge particularized to the other object. Finally, ' $N c$ ' incorrectly says that $c$ is negatively charged, and ' $N_{c} c$ ' incorrectly ascribes to $c$ a negative charge particularized to it.

Now our trope theorists can insist that it is only ever certain particularized predicates, such as ' $N_{a}$ ' and ' $N_{b}$ ', that stand for tropes. All ordinary predicates will be regarded as non-denoting. ${ }^{6}$ And some particularized predicates will be regarded as non-denoting too. Thus our trope theorists will not take a particularized predicate to stand for a trope if it doesn't apply to anything. Since $c$ is not negatively charged, there is no such thing as $c$ 's particularized negative charge so that ' $N_{c}$ ' will be regarded as non-denoting. And even if a particularized predicate does apply to something, but derives from an ordinary predicate that fails the relevant standard of naturalness, our trope theorists are still at liberty to regard it as non-denoting.

The present version of second-order trope theory thus allows for sparse tropes. Unlike in Jones' framework, where each sentence of the form $\ulcorner\exists X(X \approx \Phi)\urcorner$ and each instance (Exist2) follows logically from uncontroversial truths, nothing in the logic of the present free setting forces our trope theorists to accept such claims. Given that they regard all ordinary predicates as non-denoting, the only sentence of the form $\ulcorner\exists X(X \approx \Phi)\urcorner$ that they will ever accept are sentences of the form $\left\ulcorner\exists X\left(X \approx \Phi_{t}\right)\right\urcorner$. And even of those, they are free to only endorse the ones containing predicates that meet certain naturalness-related requirements. Similarly, they are free to only endorse those instances of

$\left(\right.$ Exist $\left._{F}\right) \quad \square \forall x\left(\Phi_{t} x \rightarrow \exists Y\left(Y x \wedge \square \forall z\left(Y z \rightarrow \Phi_{t} z\right)\right)\right)$

that feature sufficiently natural particularized predicates.

What is more, the present setting also allows us to ban free-floating tropes by endorsing:

(NFF) $\square \forall X \exists y X y$

For given the free quantifier rule $\left(\mathrm{UI} 2_{F}\right)$, (NFF) is just fine. It commits our trope theorists to the truth of $\left\ulcorner\exists y \Phi_{t} y\right\urcorner$ for each particularized predicate $\Phi_{t}$ that they take to stand for a trope (i.e. for which they accept $\left.\left\ulcorner\exists X\left(X \approx \Phi_{t}\right)\right\urcorner\right)$. It thus succeeds in ensuring that all the tropes there are have bearers, without having anything like the implausible consequence that any expressible condition is satisfied.

Finally, it becomes unproblematic to allow for contingent tropes. Our free trope theorists can regard second-order self-identity statements in general $(\ulcorner\Phi \approx \Phi\urcorner)$, and those involving particularized predicates $\left(\left\ulcorner\Phi_{t} \approx \Phi_{t}\right\urcorner\right)$ in particular, as logically true, without having to regard the result of existentially quantifying into predicate position as logically true or even as true at all. The argument to necessitist claims such as (NEC) is thus blocked, even when a standard rule of necessitation is accepted.

\footnotetext{
${ }^{6}$ Here it matters that the trope theorists are adopting a positive free second-order logic. For this allows them to regard, say, ' $N a$ ' as true while taking ' $N$ ' to be non-denoting (i.e. while taking ' $\exists X(X \approx N)$ ' to be false). A negative free second-order logic, which treats all atomic sentences containing non-denoting predicates as false, wouldn't allow for this.
} 


\subsection{Free second-order trope theory and Jones' arguments}

I take the discussion so far to show that, from a trope theoretic perspective, it is very natural to (1) adopt a second-order framework based on a positive free second-order logic and (2) flesh out this framework in such a way that only a select class of particularized predicates are taken to stand for tropes. But once the second-order framework is set up in this trope friendly way, neither of Jones' two arguments for a quick resolution in favor of universalism goes through.

Start with the comprehension argument, according to which a second-order property theory has to be universalist because all instances of the universalist comprehension schema

(Universal2) $\quad \exists X \square \forall y(X y \leftrightarrow \Phi y)$,

follow via (EG2) from uncontroversial truths of the form:

$\square \forall y(\Phi y \leftrightarrow \Phi y)$.

Since our trope-friendly second-order framework supports $\left(E G 2_{F}\right)$ instead of (EG2) the argument is blocked. All that can be derived in the present setting are truths of the form

$$
\exists X \square \forall y\left(X y \leftrightarrow \Phi_{t} y\right),
$$

for those particularized predicates $\Phi_{t}$ which are taken to stand for a trope. But since these particularized predicates only ever apply to a single object each, this does not constitute a commitment to universals.

Next, consider the same type argument, according to which second-order trope theory conflicts with there being distinct objects of the same type, which Jones takes to require true sentences of the form:

(Same Type) $\Phi a \wedge \Phi b \wedge a \neq b$

This argument is blocked because the conflict only arises against the background of (UI2). With the free $\left(\mathrm{UI} 2_{F}\right)$ in place, (Trope2) no longer entails each sentence of the form

(3) $\quad(\Phi a \wedge a \neq b) \rightarrow \neg \Phi b$

and thus no longer conflicts with (Same Type). For instance, the present version of trope theory allows for there being two things that are negatively charged $(N a \wedge N b \wedge a \neq b)$. Of course, for any particularized predicate $\Phi_{t}$ which our trope theorists take to stand for a trope, (Trope2) still entails:

$$
\left(\Phi_{t} a \wedge a \neq b\right) \rightarrow \neg \Phi_{t} b
$$

So if our trope theorists take ' $N_{a}$ ' to stand for a trope their theory entails ' $\left(N_{a} a \wedge a \neq b\right) \rightarrow \neg N_{a} b$ '. But this is unproblematic. The original conflict with (Same Type) was problematic because second-order trope theory thereby got in the way of our ordinary practice of predication. It meant that we could no longer regard ordinary predicates such as '... is negatively charged' as applying to more than one 
thing. That the present version of second-order trope theory prohibits particularized predicates from applying to more than one thing has no such troubling consequences. For it is not part of our ordinary practice of predication to apply such predicates to more than one object. Although a plausible property theory needs to preserve that $a$ and $b$ can be distinct yet both be negatively charged, it need not preserve that $a$ and $b$ can be distinct yet both have $a$ 's particularized negative charge.

\subsection{Against the argument from resolution}

The upshot of this discussion is that Jones hasn't established a general conflict between trope theory and second-order frameworks for property theories, but only a conflict between trope theory and a specific second-order framework, one which trope theorists may reject in favor of the more trope-friendly second-order framework described. I take this to show that the argument from resolution for second-order realism fails. The central claim of that argument, recall, is that while the choice between first-order universalism and first-order trope theory is a hard one, that between second-order universalism and second-order trope theory couldn't be easier, because second-order universalism turns out to be a logical consequence of uncontroversial assumptions (the comprehension argument) and second-order trope theory conflicts with basic aspects of our ordinary and scientific world-views (the same type argument). Given the availability of a second-order framework in which universalism is not a logical consequence of uncontroversial assumptions and trope theory is not in conflict with basic aspects of our world-view, this claim proves to be mistaken.

It is important to note that this conclusion is consistent with the acknowledgment that the framework recommended to second-order trope theorists comes with theoretical costs of its own. Notably, qua positive free second-order framework, it requires trope theorists to come to terms with higher-order versions of controversial phenomena characteristic of a positive free first-order framework. Just like the latter allows atomic predications $\Phi(t)$ to be true when $t$ fails to denote, the former allows $\Phi(t)$ to be true when $\Phi$ fails to denote. In each case, the proponent of the positive free approach faces the challenge of providing a semantics that underwrites the phenomenon. In addressing this challenge, the strategies available in the first-order case are also open to our trope theorists. They can employ a dual domain semantics and assign to non-denoting predicates semantic values drawn from an 'outer' domain that outruns the 'inner' domain of quantification (see e.g. Nolt 2006 for the first order-case). While this approach is controversial, recent work by Fritz and Goodman $(2016,2017)$ suggests that the most plausible higher-order contingentist theories of universals are committed to similar semantic machinery, so that we might be concerned with a commitment shared by higher-order contingentist theories of tropes and of universals. Alternatively they can try to avoid an outer domain by couching the semantics in a meta-language that is free in just the same way as the object language is (extending a strategy proposed for the first-order case by Bacon (2013) who also defends positive free logic more generally). 
To be sure, these treatments are contentious and their application to the higherorder case is under-explored. It is thus not unreasonable to regard our trope theorists' commitment to a positive free higher-order logic as a theoretical cost. For present purposes, we don't have to reach a conclusive verdict regarding its exact amount. For the present aim is not to establish that second-order trope theory is superior to second-order universalism. Rather, the aim is to show that the choice between the two options, much like that between first-order trope theory and firstorder universalism, is not a straightforward one, but one to be based on a careful and complicated cost-benefit analysis. We can agree on this without having to determine the exact theoretical costs of positive free higher-order logic, as long as we agree, as I think we clearly should, that they fall significantly short of those the argument from resolution tried to associate with second-order trope theory: whatever its exact status, it is not a 'basic aspect of our ordinary and scientific world-views' that a positive free higher-order logic is unacceptable, so that reliance on it is not a commitment that straightforwardly disqualifies a theory.

As we will see shortly, the conclusion we reached regarding the argument from resolution is also compatible with positive free second-order trope theory still facing certain problems qua trope theory, as long as these problems are sufficiently close to corresponding problems faced by first-order trope theories. I will discuss such a problem in the next section and illustrate why it cannot be used to save the argument from resolution.

\section{Russellian regresses}

The second-order trope theory developed in Sect. 3 allows for the truth of claims to the effect that distinct objects $a$ and $b$ are of the same type, where such claims are taken to have the following form:

(Same Type) $\Phi a \wedge \Phi b \wedge a \neq b$

Arguably, however, a theory which is realist about properties should allow for more than that. For even anti-realists about properties can accept truths of the form of (Same Type). Provided that electron $a$ and electron $b$ are both negatively charged, a realist theory of properties should additionally allow for the truth of some precisification of the following thesis:

(Commonality) There is something that $a$ and $b$ have in common (and $a \neq b$ ).

Universalist accounts are, of course, well equipped to do so. Thus second-order universalists will capture (Commonality) as follows:

$$
\exists X(X a \wedge X b) \wedge a \neq b
$$

Second-order trope theorists, in contrast, have a harder time. Even the more permissive framework set out in Sect. 3 does not allow them to accept (12).

This is a problem for second-order trope theory, but it is not one that serves to resurrect the argument from resolution. For that argument requires that the choice 
between trope theory and universalism be easier in the higher-order framework than it is in the first-order framework. But the present problem does not lead to any such asymmetry, since first-order trope theorists face an exact counterpart of it. While first-order universalists can capture (Commonality) with

$$
\exists x(\operatorname{Iax} \wedge \operatorname{Ibx}) \wedge a \neq b,
$$

first-order trope theorists cannot accept (13) any more than second-order trope theorists can accept (12).

At this point, a standard move by first-order trope theorists is to introduce a relational predicate, $R$, stipulated to apply to all and only those tropes that exactly resemble each other. They then propose to understand commonalities among objects as resemblance between some of their tropes, offering (14) as a precisification of (Commonality):

$$
\exists x \exists y(\operatorname{Iax} \wedge \operatorname{Iby} \wedge R(x, y)) \wedge a \neq b
$$

So far, however, there still is no asymmetry, for an analogous move at a higherorder level is available to second-order trope theorists:

$$
\exists X \exists Y\left(X a \wedge Y b \wedge R^{2}(X, Y)\right) \wedge a \neq b
$$

Here, the predicate ' $R$ ' is a second-level predicate that takes first-level predicates and second-order variables as arguments, while the first-level predicate ' $R$ ' takes singular terms and first-order variables as arguments.

Now Jones has argued, in a somewhat different context, against the viability of such resemblance based accounts in a second-order setting. ${ }^{7}$ To reach a final verdict on the argument from resolution, we need to consider whether his considerations can be used to establish the asymmetry between the first-order and second-order cases that would be needed for the argument from resolution to succeed.

To this end, suppose that not only $a$ and $b$ are negatively charged, but so is $c$. How are second-order trope theorists to account for $a, b$ and $c$ having something in common? Extending the strategy behind (15) they will offer:

$$
\exists X \exists Y \exists Z\left(X a \wedge Y b \wedge Z c \wedge R^{2}(X, Y) \wedge R^{2}(Y, Z) \wedge a \neq b \neq c\right)
$$

Note, however, that this requires that the similarity predicate ' $R$ ', can apply to two distinct pairs of second-order entities. This, Jones argues, goes against the grain of trope theory. Since he operates in a classical setting, which doesn't allow for nondenoting predicates, introducing a resemblance predicate is, for him, tantamount to introducing a resemblance relation. And his point is then that, on pain of introducing ad-hoc metaphysical distinctions, trope theorists should treat this relation as a trope

\footnotetext{
7 Jones (829-830) argues against resemblance based substitutes for statements of the form (Same Type). Since the free second-order trope theory developed in Sect. 3 allows for the truths of such statements, it doesn't need to appeal to resemblance based substitutes in that context.
} 
too (see 830). More precisely, Jones argues, they should not only endorse (Trope2), but also an analogous claim one level further up (and concerning two-place relations, rather than monadic properties) ${ }^{8}$ :

(Trope3) $\square \forall X_{1}, X_{2} \forall Y^{2}\left(Y^{2}\left(X_{1}, X_{2}\right) \rightarrow \square \forall Z_{1}, Z_{2}\left(Z_{1}, Z_{2} \not \approx X_{1}, X_{2} \rightarrow \neg Y^{2}\left(Z_{1}, Z_{2}\right)\right)\right)$

But then, Jones points out, they cannot allow ' $R$ ', to apply to distinct pairs of second-order tropes. The best they can do instead is to distinguish between two such similarity relations, $R_{1}^{2}$ and $R_{2}^{2}$, which themselves stand in resemblance relation $R^{3}$ that relates third-order entities:

$$
\exists X \exists Y \exists Z\left(X a \wedge Y b \wedge Z c \wedge R_{1}^{2}(X, Y) \wedge R_{2}^{2}(Y, Z) \wedge R^{3}\left(R_{1}^{2}, R_{2}^{2}\right) \wedge a \neq b \neq c\right)
$$

A natural worry about this strategy is then that it doesn't reach a stable resting point. Postulating the three resemblance relations, $R_{1}^{2}, R_{2}^{2}$, and $R^{3}$, allows trope theorists to account for three things having something in common, but doesn't yet allow them to account for four things having something in common. Accommodating this as well will require a further proliferation of resemblance relations. In general, unless trope theorists (implausibly) impose an upper limit on how many things can have something in common, there will likewise be no upper limit on the number of resemblance relations they need to postulate.

Note that against the background of the positive free setting developed in Sect. 3, our second-order trope theorists have a way of avoiding this proliferation of resemblance relations: they can treat the original resemblance predicate ' $R$ ' ' as nondenoting, insisting that to introduce a resemblance predicate is not to incur a commitment to a resemblance relation. In this case their endorsement of (16) no longer conflicts with (Trope3) and the whole regress argument doesn't get going. We don't need to decide here whether the regress must be stopped in the first place (or whether it is, perhaps, a non-vicious regress). We also don't have to take a definite stance on whether it is a satisfying response to let a non-denoting resemblance predicate do all the heavy lifting in a trope theorist's account of commonality claims. Our aim, recall, is not a comprehensive defense of secondorder trope theory, but a defense of the claim that the argument from resolution fails. For this purpose it suffices to show that second-order trope theory is not in any worse a position than first-order trope theory. We can thus leave it at noting that second-order trope theorists have two options when it comes to developing their resemblance based account of commonality claims: introduce a resemblance predicate without a corresponding resemblance relation, or else additionally accept the resemblance relation and come to live with the ensuing regress. As we shall see shortly, first-order trope theories have just the same options, which means that the two cases remain symmetrical, so that argument from resolution fails.

Thus, consider how first-order trope theorists will capture the claim that $a, b$ and $c$ have something in common. Extending the strategy behind (14), they will offer:

\footnotetext{
${ }^{8}$ Two comments on the notation in (Trope3): First, the variable ' $Y$ ' ' is a third-order variable that occupies the same syntactic position as the second-level predicate ' $R^{2}$ '. Second, sequences of properties are distinct $\left(Z_{1}, Z_{2} \not \approx X_{1}, X_{2}\right)$ iff their elements are (i.e. $\left.\left(Z_{1} \not \approx X_{1} \wedge Z_{1} \not \approx X_{2}\right) \vee\left(Z_{2} \not \approx X_{1} \wedge Z_{2} \not \approx X_{2}\right)\right)$.
} 


$$
\exists x \exists y \exists z(\operatorname{Iax} \wedge \operatorname{Iby} \wedge \operatorname{Icz} \wedge R(x, y) \wedge R(y, z)) \wedge a \neq b \neq c
$$

First-order trope theorists now also have two options. First, they can insist that to introduce the resemblance predicate ' $R$ ' is not to incur a commitment to a resemblance relation. Or, second, they can additionally accept the resemblance relation and face the same kind of regress argument. To see this, note that we can insist-with just the same right as before-that first-order trope theorists should treat the resemblance relation as a trope. Since first-order trope theorists treat all tropes as first-order entities, treating the resemblance relation as a trope means treating it as a first-order entity: $r$. So, really, they shouldn't leave their analysis of the claim that $a, b$ and $c$ have something in common at (18), but should offer:

$$
\exists x \exists y \exists z(\operatorname{Iax} \wedge I b y \wedge I c z \wedge I(x, y, r) \wedge I(y, z, r)) \wedge a \neq b \neq c
$$

This, however, conflicts with (a generalization of) (Trope1) as much as (16) conflicted (on the assumption that ' $R$ ' ' denotes) with (Trope3). So the best thing first-order trope theorists can do instead is to distinguish between two resemblance tropes $r_{1}$ and $r_{2}$, which themselves instantiate a further resemblance trope $r_{3}$ :

$$
\exists x \exists y \exists z\left(\operatorname{Iax} \wedge I b y \wedge I c z \wedge I\left(x, y, r_{1}\right) \wedge I\left(y, z, r_{2}\right) \wedge I\left(r_{1}, r_{2}, r_{3}\right)\right) \wedge a \neq b \neq c
$$

On this option then, the resemblance strategy doesn't reach a stable resting point in the first-order setting any more than it does in the higher-order setting. Postulating the three resemblance relations $r_{1}, r_{2}$ and $r_{3}$ allows first-order trope theorists to account for three things having something in common, but doesn't yet allow them to account for four things having something in common. Accommodating this as well will require a further proliferation of resemblance relations. In general, unless firstorder trope theorists (implausibly) impose an upper limit on how many things can have something in common, there will likewise be no upper limit on the number of resemblance relations they need to postulate.

Put in these first-order terms this objection to trope theory should sound familiar. It is, in effect, a version of Russell's regress argument for the inevitability of universals (Russell 1967: Ch. 9) applied to the specific case of tropes (as previously done by Daly 1997: 148-153). What the present section shows then is that a dialectical situation familiar from first-order trope theory carries over to secondorder trope theory: either the trope theorists make use of a resemblance predicate without accepting a commitment to a corresponding resemblance relation, or they also accept the resemblance relation and have to come to terms with a Russellian regress argument. That trope theorists face this choice regardless of whether they're operating in a first- or second-order framework is an interesting result, but not one conducive to the argument from resolution. For either the choice is sufficiently bad to discredit trope theory or it isn't. If it is, then the tropes versus universals dispute is resolved both in a first-order and in a second-order framework, in which case it is not the second-order framework that resolves it. If it isn't, then the dispute is left unresolved in both frameworks and, a fortiori, isn't resolved in the second-order framework. Either way, the argument from resolution for second-order realism fails. 


\section{Tropes versus universals: debate dissolved}

Does the failure of the argument from resolution weaken the case for second-order realism? It does weaken the specific case Jones makes for second-order realism. But I think it simultaneously points towards an alternative, and, in my view, preferable way of motivating a second-order realist position via its impact on the tropes versus universals dispute.

To see what this alternative motivation for second-order realism is, note that the natural targets for the Russellian regress arguments we encountered in Sect. 4 are what we may call pure trope theories. Impure theories, which treat some properties and relations as tropes and some others (such as the resemblance relation) as universals, easily evade the regress. Yet proponents of such regress arguments typically take them to motivate not only the rejection of a pure trope theory but also the acceptance of a pure universal theory, according to which all relations and properties are universals.

Isn't this, then, an overreaction to the regress argument? Not if one subscribes, as the proponents of such arguments seem to do, to what we may call the purity principle. According to this principle, pure property theories (which either treat all properties and relations as tropes or treat all properties and relations as universals) are, ceteris paribus, preferable to impure ones (which mix and match tropes with universals). Why accept the purity principle? The main motivation seems to be the worry that impure theories will inevitably involve arbitrary metaphysical distinctions: for instance we saw Jones arguing that there would be something objectionably ad-hoc about a trope theorist who regarded, say, charge properties as tropes while regarding the resemblance relation holding between such tropes as a universal.

At least prima facie, this worry seems well founded and the purity principle attractive. Now combine this with the observation that, again at least prima facie, there seem to be good reasons for property realists to accept universals as well as tropes. For instance, it is universals, not tropes, that are best suited for the role of properties as enablers of genuine commonalities among objects (see Sect. 4). But it is tropes, not universals, that are best suited for the role of properties as the objects of perception (as reflected in statements such as 'Olga sees the redness of the apple') or as the relata of causal relations (as reflected in statements such as 'the electron's negative charge causes it to attract protons'). ${ }^{9}$ It thus seems as if property realists find themselves in a predicament: they are faced with three prima facie attractive desiderata on a theory of properties (allow for universals, allow for tropes, make your theory pure) which cannot be jointly satisfied. Part of the job of any property realist would then appear to be that of deciding which desideratum to reject.

However, I think that the distinction between first-order and second-order realism can be used to show that we are here concerned with another instance of a

\footnotetext{
9 On the role of tropes in perception see e.g. Williams (1953: 16-17), Campbell (1981: §4), Mulligan et al. (1984), Mulligan (1999), Lowe (1998: 204-205) and Nanay (2012). On the role of tropes in causality see e.g. Williams (1953: 172), Campbell (1990: 22-23) and Ehring (1997: 71-90, 2011: 136-172).
} 
theoretical choice which is only apparently forced upon property realists and is in fact not as inevitable as it may first seem. For once we distinguish between firstorder and second-order realism we should distinguish between two ways to understand the purity principle. On a weaker reading, all it demands is intra-level purity: no theory should mix first-order tropes with first-order universals or secondorder tropes with second-order universals. On a stronger reading, it additionally demands inter-level purity: no theory should mix first-order tropes with secondorder universals or first-order universals with second-order tropes.

With these two notions of purity in mind, consider a view-hybrid realismwhich is realist about tropes and about universals, while taking tropes to be firstorder entities but universals to be second-order entities. Thus hybrid realism's realism about tropes is adequately expressed by (Exist1) and (Trope1) and its realism about universals is adequately expressed by (Exist2) and (Universal2).

Hybrid realism countenances universals and therefore enjoys benefits typically associated with them. Thus it has an easy time accounting for the role of properties as enablers of genuine commonalities among objects. Since it is a universal theory at the second-order level, it can straightforwardly accept as true not only sentences such as ' $N a \wedge N b \wedge a \neq b$ ' but also the results of existentially quantifying into the predicate position, such as ' $\exists X(X a \wedge X b \wedge a \neq b)$ '.

Hybrid realism also countenances tropes and therefore also enjoys benefits typically associated with them. Thus it has an easy time accounting for the role of properties as the objects of perceptions and the relata of causal relations. Since it is a trope theory at the first-order level, it provides tropes as the referents for the property designators in 'Olga sees the redness of the apple' and 'the electron's negative charge causes it to attract protons'.

Does hybrid realism also satisfy the purity desideratum? The answer is no longer trivial. While it may initially have looked as if any theory satisfying two of the desiderata would eo ipso have to flout the third, this is no longer clear. For while hybrid realism doesn't exhibit inter-level purity (it mixes first-order tropes with second-order universals), it does exhibit intra-level purity (it doesn't mix tropes and universals on any one level). Is this enough purity to count as satisfying the purity desideratum? I think it is. It was an endeavor to avoid arbitrary metaphysical distinctions that motivated the purity desideratum in the first place, so the relevant question is how well intra-level purity serves this purpose. And the answer is: pretty well. For instance, it would clearly be objectionable to treat, say, the first-order property of weighing $5 \mathrm{~kg}$ as a trope while treating the first-order property of being red as a universal. But mixing tropes with universals in such problematic ways is already ruled out by intra-level purity. In contrast, the way in which hybrid realism is mixing tropes and universals is not arbitrary at all: the line it is drawing between tropes and universals is that between first-order and second-order quantification. Given that second-order quantification is assumed to be a sui generis quantification from the outset (see Sect. 1) this is a line as purely logico-metaphysical and jointcarving as one could possibly ask for.

What is more, the hybrid realist can effectively diffuse the feeling that her position lacks purity. To see how, we can bring to bear a helpful observation made by Jones in the course of his defense of second-order realism. He points out that the 
natural language sentence 'there are properties' might not be an ideal vehicle to communicate second-order realism, since it would appear to be best formalized with the help of a first-order existential quantifier, and a predicate 'property( )' whose argument position accepts first-order variables. What would be needed instead is a second-order analogue of this predicate, 'PROPERTY ${ }^{2}()$ ', whose argument position accepts second-order variables (817). In the same way, we can distinguish between a pair of predicates, 'trope( )' and 'universal( )', whose argument positions accept firstorder variables, and their second-order analogues, 'TROPE ${ }^{2}\left(\right.$ )' and 'UNIVERSAL ${ }^{2}($ )'. With these distinctions in play, hybrid realism's (intra-level) purity manifests itself in its rendering the following two claims true:

$\begin{array}{ll}\text { (Purity } 1) & \forall x(\operatorname{property}(x) \rightarrow \operatorname{trope}(x)) \\ \text { (Purity2) } & \forall X\left(\operatorname{PrOPERTY}^{2}(X) \rightarrow \operatorname{UNIVRSAL}^{2}(X)\right)\end{array}$

These principles ensure that all properties are of one kind, and that all PROPERTIEs ${ }^{2}$ are of one kind. The impression that hybrid realism is still lacking in purity is likely to be grounded in the idea that one could intelligibly require a theory to additionally ensure that properties and PROPERTIES $^{2}$, taken together, are of one kind, by ensuring the truth of something like 'everything that is a property or a PROPERTY ${ }^{2}$ is $F$ '. But there is no coherent such request to made: in contrast to (Purity1) and (Purity2) the precise formulation of this alleged further expression of purity would require what cannot be done, namely to simultaneously bind the first-order variable in 'property $(x)$ ' and the second-order variable in 'PROPERTY ${ }^{2}(X)$ '.

The hybrid realist is exploiting here an insight that goes back to Frege, commonly regarded as the first proponent of a higher-order conception of properties. Thus Frege famously states with respect to his distinction between objects (his term for first-order entities) and concepts (his term for certain higher-order entities) that:

I do not want to say it is false to say concerning an object what is said here concerning a concept; I want to say it is impossible, senseless to do so. (Frege 1892: 189)

The hybrid realist is bringing this insight to bear on the case at hand: since neither first-level predicates, such as 'property( )' or 'trope( )', nor second-level predicates, such as 'PROPERTY ${ }^{2}\left(\right.$ )' or 'UNIVERSAL ${ }^{2}()$ ', can be intelligibly applied to both firstorder and second-order entities, an additional request for purity of the form considered is bound to be unintelligible, or as Frege would have put it, senseless.

In this way, the distinction between first-order and second-order realism can be used to dissolve, rather than to resolve, the tropes versus universals dispute. Just like this distinction revealed the transcendentalism versus immanentism dispute as based on a false dilemma (see Sect. 2), it reveals the tropes versus universals dispute as based on a false trilemma: hybrid realism allows for tropes and universals while still meeting the purity requirement. And just like the dissolution of the transcendentalism versus immanentism dispute results from the second-order realist's rejection of the question after the location of (second-order) properties as unintelligible, the dissolution of the tropes versus universals dispute results from the hybrid realist's rejection of certain requests for additional purity as unintelligible. 


\section{Dissolution defended}

I will close by addressing three objections to the claim that hybrid realism dissolves the tropes versus universals debate.

The first objection notes that, in Sect. 4, we saw Jones relying on purity considerations when arguing that a theory which treats second-order entities as tropes (and thus endorses (Trope2)) should do the same with respect to third-order entities (and thus also endorse (Trope3)). Isn't Jones concerned here with an intelligible notion inter-level purity? If so, how does this fit together with the considerations from the last section and does hybrid realism exhibit the purity Jones is looking for?

In response, note that Jones cannot be requiring a theory to ensure the truth of something like 'everything that is a PROPERTY ${ }^{2}$ or a PROPERTY ${ }^{3}$ is $F$ '. As before, there is no intelligible such request to be made, this time because it would require simultaneous binding of second-order and third-order variables. What Jones is requiring, rather, is that if a property theory makes claims typical for tropes(/ universals) on any one higher-order level, then it ought to make analogous claims on each higher-order level. This is an intelligible request but it is one that hybrid realism meets, or rather, will meet once it is appropriately developed. So far, we have focussed exclusively on the first-order and second-order level and have characterized hybrid realism as the combination of first-order tropes (as reflected by its endorsement of (Trope1)) with second-order universals (as reflected by its endorsement of (Universal2)). The natural way to round out this theory is by taking it to additionally endorse, for each $n \geq 1$, the resulting instance of the typically ambiguous schema:

(Universal $\left.{ }_{H O}\right) \quad \exists X^{n+1} \square \forall Y^{n}\left(X^{n+1} Y^{n} \leftrightarrow \Phi Y^{n}\right)$

Thus developed, hybrid realism exhibits the kind of purity Jones is after, namely by treating all higher-order entities as governed by analogous universalist principles.

The second objection follows up on the first and maintains that a theory-strict universalism - which endorses all the same instances of $\left(\right.$ Universal $\left._{H O}\right)$ but combines this with (Universal1), rather than with (Trope1), exhibits even more theoretical uniformity than hybrid realism. It takes this to show that hybrid realism cannot do full justice to the purity principle and therefore cannot be seen as dissolving the need to choose between tropes, universals and purity.

In response, first note that it is not clear that we should grant the objection's assumption that strict universalism exhibits, overall, more theoretical uniformity than hybrid realism. For (1) it is not clear how much more inter-level uniformity is really afforded by swapping (Universal1) for (Trope1): the schemas resulting from (Universal $_{H O}$ ), such as e.g. those for $n=1$ and $n=2$, clearly resemble each other much more than they resemble (Universal1) which involves an instantiation relation absent from any instance of (Universal $\mathrm{HO}_{\mathrm{O}}$ ). And (2) even if strict universalism was credited with slightly more inter-level uniformity than hybrid realism, this may be offset by a loss of intra-level uniformity. Thus, according to hybrid realism, it is true that every first-order entity is unrepeatable: neither tropes nor any other first-order entities (such as e.g. ordinary concrete objects) can be multiply instantiated. This 
generalization about first-order entities does not hold according to strict universalism with its multiply instantiable universals on the first-order level. A potential increase in inter-level uniformity on part of strict universalism thus comes at the cost of reduced intra-level uniformity on the first-order level, rendering its claim to more overall uniformity questionable.

Second, note that to dissolve the tropes versus universals dispute in the way described it is not necessary that hybrid realism be a theory than which no more unified theory can be conceived. All that is required is that it be sufficiently uniform to do justice to the motivation behind the purity principle, i.e. sufficiently uniform to avoid arbitrary metaphysical distinctions. And while it would be arbitrary to treat, say, 14th level properties as tropes, while endorsing universalist principles on all other levels, no such arbitrariness afflicts hybrid realism's distinction between the base-level on the one hand and all other levels on the other. The situation can be instructively compared to the recent debate about combinations of necessitist and contingentist claims of various orders: here, too, the combination of contingentism on the $14^{\text {th }}$ level with necessitism on all other levels would be arbitrary, while the combination of contingentism on the first-order level with necessitism on all higherorder levels clearly isn't arbitrary in the same way (see Goodman 2016: $\$ 1.1$ and Williamson 2016: 640-641). In fact, some first-order versus higher-order asymmetries are to be expected. For, as Goodman (614) points out, (1) the hierarchy of types of a formal higher-order language constitutes an inductively defined class with the type of first-order entities serving as the base case and (2) when it comes to such classes, it is perfectly normal that the base case fails to satisfy some generalizations that the remaining members do satisfy (think, e.g., of the class of natural numbers as inductively defined with 0 as the base case).

The third objection grants that the distinction between first-order and higherorder realism can effectively dissolve the tropes versus universals dispute in a way similar to how it can dissolve the transcendentalism versus immanentism dispute. But it questions whether both dissolutions can be had simultaneously. For it notes that hybrid realism, by incorporating first-order realism (about tropes), re-introduces the possibility of posing intelligible questions about the location of properties, even if it is granted that locational vocabulary can only be combined with singular terms.

In response, first note that even if the proposed dissolution of the tropes versus universals debate turned out to be in tension with the dissolution of the immanentism versus transcendentalism debate, its availability would still be an interesting result, and one conducive to the general aim of showing how higherorder conceptions of properties can fruitfully reshape traditional debates about the nature of properties. For instance, it would make a second-order realist position attractive for those who are unimpressed by a dissolution of the immanentism versus transcendentalism debate, perhaps because they took this debate to be easily decided anyway.

However, I think that in an important sense, the two dissolutions are compatible. For while hybrid realism is realist about first-order properties, and therefore allows us to ask about their location, it treats all of these first-order properties as tropes, and thus as unrepeatable entities. While hybrid realism thus re-introduces the possibility 
to pose intelligible questions concerning the location of certain properties, it also allows for relatively easy answers to these questions: since each trope has exactly one bearer it is unproblematic to say that tropes are wherever their bearers are. What makes the corresponding stance about the location of universals so problematic is that universals, unlike tropes, can be multiply instantiated, thereby requiring us to say things often taken to be mysterious, such as that they are, at once, wholly located in distinct places (see e.g. Lowe 2004: 23-25). So it is not so much questions about the location of properties that it would be nice to avoid but questions about the location of universals. And hybrid realism still allows us to do that: since it treats all universals as higher-order entities it remains entitled to regard questions concerning their location as unintelligible. It thus avoids the obligation to engage with location questions in precisely those cases where this is most beneficial.

\section{Conclusion}

I've argued for two main claims. The first is that the argument from resolution for a higher-order conception of properties fails because this conception does not in fact lead to a resolution of the tropes versus universals dispute in favor of universals. To establish this claim, I have developed a novel formulation of second-order trope theory and shown it to be immune to anti-trope-theoretic arguments specific to the second-order setting. While this second-order trope theory still faces certain problems, these mirror corresponding problems faced by first-order trope theories, so that they don't serve to resurrect the argument from resolution which crucially requires the choice between universals and tropes to be easier in a second-order setting than it is in a first-order setting.

The second main claim I have argued for is that a higher-order conception of properties can nevertheless be motivated by its impact on the tropes versus universals debate. For a higher-order conception of universals can be combined, in the form of hybrid realism, with a first-oder trope theory in a way that dissolves, rather than resolves, the tropes versus universals disputes: hybrid realism combines the advantages of both tropes and universals while avoiding the charge of drawing unprincipled metaphysical distinctions. Hybrid realism's dissolution of the dispute is reflected in the theory's allowing us to say, simultaneously, that all properties are tropes (if 'property' is taken to apply to first-order entities) and that all properties are universals (if 'property' is taken to apply to higher-order entities), thereby undermining the need to chose between tropes and universals in the first place, a result that I have defended against several objections.

Acknowledgements I would like to thank the audiences at the Joint Sessions 2020 at the University of Kent and at the research colloquium Sprache und Welt at the University of Hamburg for helpful discussion. Many thanks are also due to Fiona Doherty, Rob Trueman, and Richard Woodward as well as to an anonymous referee for this journal for helpful comments that improved the paper in many ways.

Open Access This article is licensed under a Creative Commons Attribution 4.0 International License, which permits use, sharing, adaptation, distribution and reproduction in any medium or format, as long as you give appropriate credit to the original author(s) and the source, provide a link to the Creative 
Commons licence, and indicate if changes were made. The images or other third party material in this article are included in the article's Creative Commons licence, unless indicated otherwise in a credit line to the material. If material is not included in the article's Creative Commons licence and your intended use is not permitted by statutory regulation or exceeds the permitted use, you will need to obtain permission directly from the copyright holder. To view a copy of this licence, visit http:// creativecommons.org/licenses/by/4.0/.

Funding Open Access funding enabled and organized by Projekt DEAL.

\section{References}

Bacon, A. (2013). Quantificational logic and empty names. Philosophers' Imprint, 13(24), 1-21.

Bacon, A., Hawthorne, J., \& Uzquiano, G. (2016). Higher-order free logic and the Prior-Kaplan paradox. Canadian Journal of Philosophy, 46(4-5), 493-541.

Besson, C. (2009). Externalism, internalism, and logical truth. The Review of Symbolic Logic, 2(1), 1-29.

Campbell, K. (1981). The metaphysic of abstract particulars. Midwest Studies in Philosophy, 6(1), 477-488.

Campbell, K. (1990). Abstract particulars. Oxford: Blackwell.

Correia, F. (2006). Generic essence, objectual essence, and modality. Noûs, 40(4), 753-767.

Correia, F., \& Skiles, A. (2019). Grounding, essence and identity. Philosophy and Phenomenological Research, 98(3), 642-670.

Daly, C. (1997). Tropes. In D. H. Mellor \& A. Oliver (Eds.), Properties (pp. 140-159). Oxford: Oxford University Press.

Dorr, C. (2016). To be F is to be G. Philosophical Perspectives, 30(1), 39-134.

Ehring, D. (1997). Causation and persistence: A theory of causation. Oxford: Oxford University Press.

Ehring, D. (2011). Tropes: Properties, objects, and mental causation. Oxford: Oxford University Press

Frege, G. (1892). On concept and object. In M. Beaney (Ed.), The Frege reader (pp. 181-93). Oxford: Blackwell.

Fritz, P., \& Goodman, J. (2016). Higher-order contingentism, part 1: Closure and generation. Journal of Philosophical Logic, 45(6), 645-695.

Fritz, P., \& Goodman, J. (2017). Counting incompossibles. Mind, 126(504), 1063-1108.

Goodman, J. (2016). Williamson on necessitism. Canadian Journal of Philosophy, 46(4-5), 613-639.

Jones, N. (2018). Nominalist realism. Noûs, 52(4), 808-835.

Jones, N. (2019). Propositions and cognitive relations. Proceedings of the Aristotelian Society, 119(2), $157-78$.

Lewis, D. (1983). New work for a theory of universals. The Australasian Journal of Philosophy, 61(4), 343-377.

Lowe, E. J. (1998). Possibility of metaphysics. Oxford: Oxford University Press.

Lowe, E. J. (2004). The four-category ontology: A metaphysical foundation for natural science. New York: Oxford University Press.

Mulligan, K. (1999). Perception, particulars and predicates. In D. Fisette (Ed.), Consciousness and intentionality: Models and modalities of attribution (pp. 163-194). Dordrecht: Kluwer.

Mulligan, K., Smith, B., \& Simons, P. (1984). Truth-makers. Philosophy and Phenomenological Research, 44(3), 278-321.

Nanay, B. (2012). Perceiving tropes. Erkenntnis, 77(1), 1-14.

Nolt, J. (2006). Free logic. In D. Jaquette (Ed.), Philosophy of logic (pp. 1023-1060). Amsterdam: Elsevier.

Rayo, A. (2013). The construction of logical space. Oxford: Oxford University Press.

Russell, B. (1967). The problems of philosophy. Oxford: Oxford University Press.

Stalnaker, R. (2012). Mere possibilities: Metaphysical foundations of modal semantics. Princeton: Princeton University Press.

Trueman, R. (2018). The prenective view of propositional content. Synthese, 195(4), 1799-1825.

Trueman, R. (2020). Idealism and the identity theory of truth. Mind. https://doi.org/10.1093/mind/fzz084.

Trueman, R. (forthcoming). Properties and propositions: The metaphysics of higher-order logic. Cambridge: Cambridge University Press. https://www.cambridge.org/de/academic/subjects/ 
philosophy/epistemology-and-metaphysics/properties-and-propositions-metaphysics-higher-orderlogic?format=HB.

Williams, D. C. (1953). The elements of being. Rev. Metaphys., 7(1-2), 3, 171-18, 192.

Williamson, T. (2003). Everything. Philosophical Perspectives, 17(1), 415-465.

Williamson, T. (2013). Modal logic as metaphysics. Oxford: Oxford University Press.

Williamson, T. (2016). Reply to Goodman. Canadian Journal of Philosophy, 46(4-5), 640-653.

Publisher's Note Springer Nature remains neutral with regard to jurisdictional claims in published maps and institutional affiliations. 